\title{
BUILDING INFORMATION MODELLING OF A MULTI STOREY BUILDING USING TERRESTRIAL LASER SCANNER AND VISUALISATION USING POTREE: AN OPEN SOURCE POINT CLOUD RENDERER
}

\author{
Yeshwanth Kumar A. ${ }^{1 *}$, Noufia M. A. ${ }^{1}$, Shahira K. A. ${ }^{1}$, A. M. Ramiya ${ }^{1}$ \\ ${ }^{1}$ Dept. of Earth and Space Sciences, Indian Institute of Space Science and Technology - \\ yeshwant.sc18m031@pg.iist.ac.in, staryesh@gmail.com
}

Commission II

KEY WORDS: Building Information Modelling, BIM, Terrestrial Laser Scanner, Lidar, Potree, Point Cloud, FARO Focus S 350

\begin{abstract}
:
With the rapid development in infrastructure, the need to document man-made structures is in increasing demand and inevitable. Such a process of digital documentation of buildings is called Building Information Modelling (BIM). Conventional techniques of BIM involve manual drafting \& modelling using computer aided design, drafting \& modelling software. Although these techniques are more accurate, given the increase in the size and complexity of modern structures, it would be tedious and time consuming for such manual work. It is in this context LiDAR shows great potential to simplify this task. Laser scanning enables rapid mapping of a building with a high degree of spatial accuracy. Since the spatial point sampling distance of any LiDAR scanner is usually in the order of centimetres or millimetres, this has potential not only to generate high density scans of the building but also to identify even the smallest defects in a structure. This facilitates using LiDAR to study the serviceability of a building. In this project, the feasibility of using a terrestrial laser scanner (TLS) to scan a multi-storey building was investigated. Additionally, the reliability of Potree for visualising point cloud data was tested. Potree is an open-source WebGL based point cloud renderer. Potree enables us to render point clouds and visualise in a portable web application. This application is also capable of making measurements of high accuracy on the 3D model of the library. This could serve to be of great utility in surveying applications. The object of study was chosen as a six-storey building, each floor having differing layouts. Two of these storeys were below ground surface level which also proved to be a test for the reliability of TLS in challenging terrain. The building has a towering height and large footprint which made it a perfect candidate for this project. A total of 54 scans (44 interior scans and 10 exterior scans of the library) were acquired with each subsequent scan station not more than $10 \mathrm{~m}$ apart from the previous one. This data was brought to the lab for further processing. The processing was carried out using open-source software packages (LAStools, CloudCompare, etc). After processing, the complete point cloud data had 483,292,994 points. In order to make the data easier to handle, spatial sub-sampling of the data was done after which the final point cloud had 87,789,548 points. Finally, this sub-sampled point cloud was published using the open source Potree Converter into an interactive web application.
\end{abstract}

\section{INTRODUCTION}

\subsection{Building Information Modelling}

In this modern era, the burgeoning of infrastructure has impacted the entirety of the globe. With such a rapid boom in infrastructure projects, it becomes increasingly important to document the schematics of these projects. This is especially important in developing nations like India, where the need for improved infrastructure often necessitates documentation of existing structures and facilities. In earlier days, such documents were prepared by means of manual drafting which was time consuming, tedious and prone to error. With recent advancements in computer aided drafting, digital drafting and documentation of these buildings has greatly reduced the time consumption of this process. In this regard, one of the newer approaches is to construct a complete information model of a structure, called a Building Information Model (BIM). Building information modelling is one of the promising recent developments in the architecture, engineering and construction (AEC) industry. With BIM technology, an accurate virtual model of a building can be digitally constructed (Azhar, 2011).

When a BIM is completed, these computer generated models

\footnotetext{
${ }^{*}$ Corresponding author
}

contain precise geometry and data needed to support the construction, fabrication and procurement activities through which the building is realised (Eastman et al., 2011). BIMs are normally developed in the conceptualisation phase of large construction projects. This application of BIMs has greatly catalysed the decision making process between client calls in such projects, where manual drafts would mean long periods of revisions between client meetings. In addition to this, BIMs are also generated for existing structures for the purpose of digital documentation and archiving, heritage mapping, visualisation, etc. In contrast to conventional manual drafting methods of building documentation, a BIM is much more easy to understand and intuitive for people from non-technical backgrounds. This means that demonstration of a construction proposal to potential customers and investors is much more easier with a BIM than with traditional methods. Due to the aforementioned properties and advantages, building information models have increasing potential and applications in the architecture, engineering and construction industry.

\subsection{Light Detection and Ranging (LiDAR)}

Light Detection and Ranging (LiDAR) is a technique of remote sensing which can be used to obtain a three dimensional point cloud of any object. The working principle of a LiDAR is very 
much similar to RADAR, except in that it uses optical light waves instead of radio waves. In a LiDAR, a laser beam is sent out which is then received after being reflected by the target. Since the time interval between emission and receiving of light is recorded, the distance of the target point from the LiDAR instrument can be calculated using the following expression:

$$
d=\frac{c t}{2}
$$

where $\quad d=$ distance between scanner and target point

$c=$ velocity of light in air

$t=$ time travelled by laser beam

LiDAR offers great potential in three dimensional remote sensing of real world objects. With the recent advancements in LiDAR technology, the time-consuming days of mapping by hand seem numbered, as geographic information system (GIS) experts, professional land surveyors (PLS) and others within the field of cartography awaken to the advantages of integrating light detecting and ranging into their mobile mapping systems (Schwarz, 2010).

\subsection{Terrestrial Laser Scanning}

LiDAR scanning systems can be broadly classified as aerial and terrestrial scanning systems. Both of these systems have their inherent advantages and disadvantages. Aerial Laser Scanners (ALS) offer wide coverage of a large area, although they are only able to map the top surface of any landscape and unable to provide comprehensive ranging information of the underlying targets. Terrestrial Laser Scanners (TLS) on the other hand offer comprehensive coverage of targets with minimal occlusion (with multiple scan stations). However, they are stationary and only able to cover a small region at a time. With regard to these advantages and limitations of each system, choosing an optimal scanning method depends on the particular requirements of the study where the LiDAR system is to be used.

In light of the ability of TLS to cover a target comprehensively with minimal occlusion, terrestrial laser scanning approach would best serve the task of building information modelling. With the developed laser scanners, the control software and software for model generation, very powerful tools are available that are suitable for most industrial and cultural heritage surveying tasks (Fröhlich, Mettenleiter, 2004). Nowadays, terrestrial laser scanning has proven to be an efficient approach for highly detailed acquisition of building facades. But the problems of slow acquisition and manual registration remain bottlenecks in the slow scanning procedure (Pu, Vosselman, 2009).

\section{OBJECTIVES}

For the purpose of this project, the general objective was to create a simple building information model of a multi storey structure for visualisation and digital documentation. This general objective can be arrived at by means of accomplishing the following specific objectives:

1. Obtaining a high density comprehensive point cloud representation of the structure by means of multiple terrestrial laser scans.

2. Generating the building information model for visualisation using Potree: an open source WebGL based point cloud renderer.

\section{MATERIALS AND METHODS}

\subsection{Object of Study}

For the purpose of this project, the object of study was chosen as the library of the Indian Institute of Space Science and Technology. It is a six storey structure which each floor having differing layout. The complex and modern architecture of the building made it an ideal target for our project and it is shown in Figure 1.

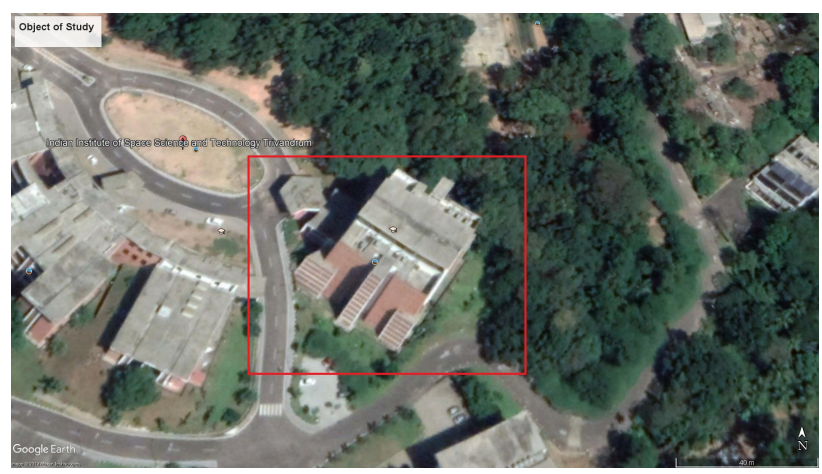

Figure 1. Google Earth imagery of the object of study (IIST Library).

\subsection{TLS Data}

In order to scan the library completely, a terrestrial laser scanner was used. A total of 54 scans were carried out to cover both the exterior and interior of the library. The scanner of choice for this purpose was the FARO Focus S 350. Initially, a reconnaissance survey was carried out to identify the scan route to be followed. While scanning the exterior of the library, each successive scan station was placed within $20 \mathrm{~m}$ from the previous scan. Alternatively, when scanning the interior of the library, each successive scan station was placed within $10 \mathrm{~m}$ from the previous scan due to increased probability of occlusion indoors. In the past, this sensor has been used in studying the performance of low rise construction under wind and coastal hazards during the landfall of hurricane Harvey near Rockport, Texas, USA (Kijewski-Correa et al., 2018). The technical specifications of the sensor are presented in Table 1.

\begin{tabular}{|l|c|}
\hline Property & Specification \\
\hline Range & 0.6 to 350 metres \\
Angular FOV (vertical) & $300^{\circ}$ \\
Angular FOV (horizontal) & $360^{\circ}$ \\
Laser Class & Class I \\
Laser Wavelength & $1550 \mathrm{~nm}$ \\
Beam Divergence & $0.3 \mathrm{mrad}$ \\
Beam Diameter at Exit & $2.12 \mathrm{~mm}$ \\
\hline
\end{tabular}

Table 1. Technical Specifications of FARO Focus S 350.

After scanning the entire library (interior and exterior), the resulting point cloud data was found to have 483,292,994 points. The points in this point cloud also had spectral (RGB) information associated with them since this scanner also enables the capture of spectral information by means of a high dynamic range (HDR) capable camera of up to 165 megapixels.

\subsection{Methods}

The workflow for this project consisted of three major steps: 
1. Acquisition of point cloud data from terrestrial laser scanning of the library.

2. Registration and processing of the point cloud data.

3. Visualising the 3D model of the library using the Potree point cloud renderer.

The processes involved in these steps are explained in detail in the following sections and an overall outline of these processes are illustrated in Figure 2.

3.3.1 Reconnaissance Survey: In order to acquire TLS scans of the library, it was first necessary to identify the optimal scanning path and most suitable scanning locations. Therefore, a reconnaissance survey of both the exterior and interior of the library was conducted during which the scanning path was identified. Along the identified scanning path, 10 suitable scanning locations were identified in the exterior and 44 suitable scanning locations were identified in the interior of the library. The reconnaissance survey was carried out over the duration of one day.

3.3.2 Preparing the instrument: After identifying the scan locations, it is necessary to calibrate the instrument to the scan location conditions. The profiles were set up in such a way that the maximum distance between two successive scans was $20 \mathrm{~m}$ outdoors and $10 \mathrm{~m}$ indoors. At each scan station, the instrument was levelled using the built in dual axis compensator in the instrument. The scan FOV was set to $300^{\circ}$ in the vertical (full FOV) and $360^{\circ}$ in the horizontal (full FOV).

3.3.3 Acquisition of Terrestrial Laser Scans: Upon proper calibration of the instrument, the TLS survey was commenced as per the pre-determined survey path. At each survey station, the instrument was placed, levelled and the point cloud was recorded. Between each scan station, it was ensured that there was good amount of overlap in order to make the registration process more convenient. Furthermore, ensuring good amount of overlap between scans would also minimise the occlusion caused by stationary and moving obstacles. The survey of the entire library took around 13 man hours to complete. When the instrument is continuously operated, it was noted that it tends to overheat. Therefore, it was necessary to introduce breaks in between the survey to ensure the instrument was at safe temperatures while operating. At the end of the survey, the change in lighting conditions from day to night could have been a probable cause for the inconsistency in the spectral features of some scans.

3.3.4 Registration of Scans: After completing the TLS survey of the library, point cloud data from 54 scans was obtained which was imported into the FARO Scene 2018 software. Here, individual point clouds were subjected to minor pre-processing such as colourisation of scans, filtering dark scan points, points beyond a distance threshold from scanner, stray points, finding planes and spheres for registration, etc. After the initial preprocessing, the scan point clouds were manually registered one to one sequentially in the original order of acquisition of scans. Between each point cloud pair, tie points and planes present in both scans were manually selected to register the pair together. The selection of these tie features was iteratively updated until the registration results were satisfactory. This process was carried out for every sequential point cloud pair. At the end of manual registration, the resulting point cloud was optimised again using the Cloud to Cloud Optimisation feature. Finally, 
the registered point cloud was visualised in the software and checked for any errors in the registration. This entire process was repeatedly carried out until the registration results were acceptable with minimal errors (FARO Scene 2018.0.0.648, 2018).

Finally, after successfully registering the individual scans into a single point cloud, the resulting point cloud was exported from the proprietary FARO Laser Scan (.fls) file format to a general ascii file format (.xyz).

3.3.5 Converting to LAS/LAZ format: At the end of the registration process, the point cloud was exported to an ascii file format. Although this file format is suitable for most of the further processing steps, the large file size of the ascii file format would prove to be a limiting factor in these steps. Furthermore, different software and sensors may use different ascii file formats which could lead to ambiguity and unintended data loss when working between multiple software for processing. In order to overcome this, the resulting point cloud would have to be converted to a uniform file format that is inter-operable between multiple software and sensors. The ASPRS LAS file format is one such file format. The major advantages of the ASPRS file format are as follows (Samberg, 2007):

- It is already recognised and accepted as an industry lidar standard worldwide.

- Allows to speed up entire lidar data processing and project management processes.

- It is a binary file format which allows to significantly reduce space required for lidar data storage.

In addition to converting to LAS file format, the point cloud data was further compressed using the LAZ file format which is a highly efficient, lossless point cloud compression scheme (Isenburg, 2013). This was done using the LAStools software suite (Isenburg, 2012).

3.3.6 Spatial sub-setting and sub-sampling: After compressing the point cloud in LAZ file format, it was imported into the CloudCompare software. Although prior to registration of the scans, the distant points were removed using a filter, some distant points would still remain in the registered point cloud. Also, there may be a lot of points from background sources apart from the object of study such as ground, clouds, vegetation, other structures, etc. These points will need to be removed from the point cloud in order to reduce redundant processing of these points.

After sub-setting the point cloud, it was now necessary to normalise the density of the point cloud. Since the original point cloud would normally have varying density, regions with high density would require more computational resources for processing. In order to minimise this, the point cloud is downsampled to a more optimal density. These processes of spatial sub-setting and sub-sampling were accomplished using the CloudCompare software (Girardeau-Montaut, 2016).

3.3.7 Cleaning of the sub-sampled point cloud: Once sub-sampling of the point cloud is complete, it should be noted that the point cloud still contains potentially unwanted and noisy points. These points could be referred to as 'outliers' and need to be removed from the point cloud. The statistical outlier removal requires two parameters:
1. Number of points to use for mean distance estimation $(N)$

2. Standard deviation multiplier threshold $\left(n_{\sigma}\right)$

After assigning these parameters, the maximum distance $\left(d_{\max }\right)$ is calculated from the following expression:

$$
d_{\text {max }}=d_{\text {avg }}+n_{\sigma} \sigma
$$

where $\quad d_{\text {avg }}=$ mean distance from point to neighbours $\sigma=$ standard deviation of neighbourhood

In this filter, for every point, $N$ nearest neighbours are identified and the average distance of the point from these neighbours is computed. If this average distance is greater than the maximum distance computed from the above expression, then the point is an outlier and removed. Otherwise, the point is retained. At the end of this algorithm, the resulting point cloud will be free of outliers and ready for further processing. The process of statistical outlier removal was also carried out using the CloudCompare software (Girardeau-Montaut, 2016).

3.3.8 Computing normals: Additionally, it may be useful to compute the normals of each point in the point cloud to correct any erroneous surfaces in the cloud. Once again, this was done using the CloudCompare software (Girardeau-Montaut, 2016). While computing normals, the local surface model was set to 'Triangulation' since this model yields better results at sharp corners and edges, although with more susceptibility to noise. After computing the normals, they were converted to HSV colors to visualise the points with different orientations as shown in Figure 3.

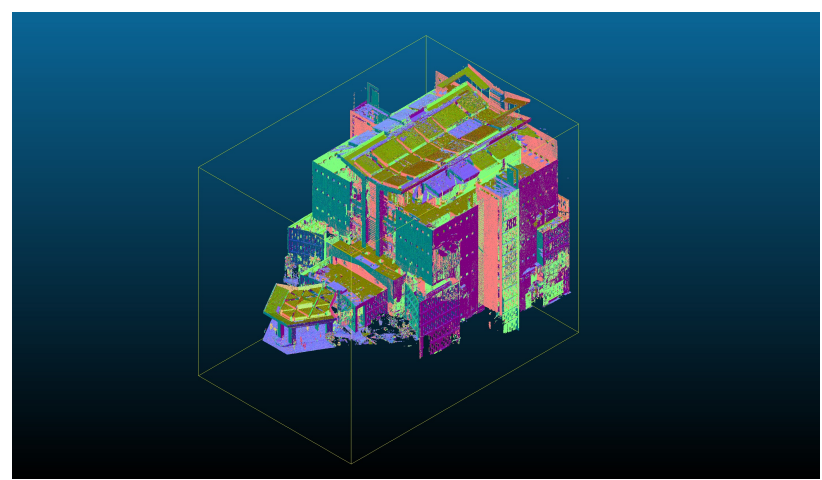

Figure 3. Normals computed for each point and converted to HSV space.

3.3.9 Publishing the point cloud using Potree: Finally, after the processing of the point cloud is completed, it is imported into the Potree application. Potree is a web-based renderer for large point clouds. It allows users to view data sets with billions of points, from sources such as LIDAR or photogrammetry, in real time in standard web browsers. One of the main advantages of point cloud visualization in web browser is that it allows users to share their data sets with clients or the public without the need to install third-party applications and transfer huge amounts of data in advance (Schuetz, 2016).

Using Potree, the final point cloud was rendered and visualised as a web application in a normal browser. 


\subsection{RESULTS}

Upon publishing the point cloud using Potree, the end product was a WebGL based point cloud renderer, that could be used to visualise the processed point cloud in any web browser as shown in Figure 4.

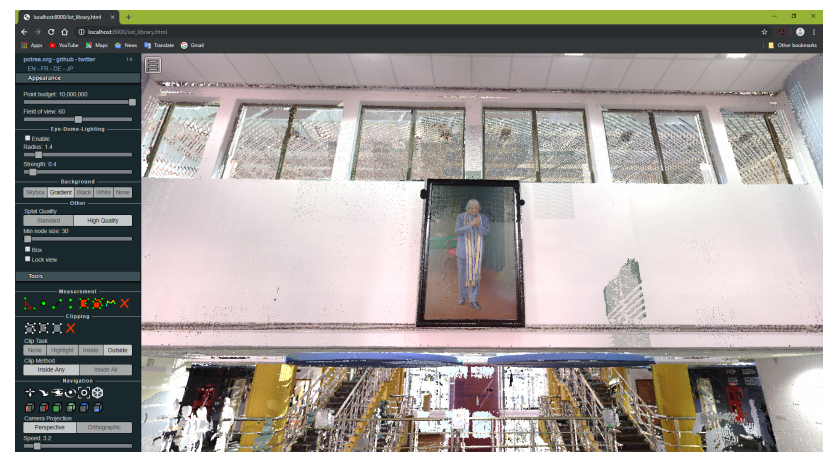

(a)

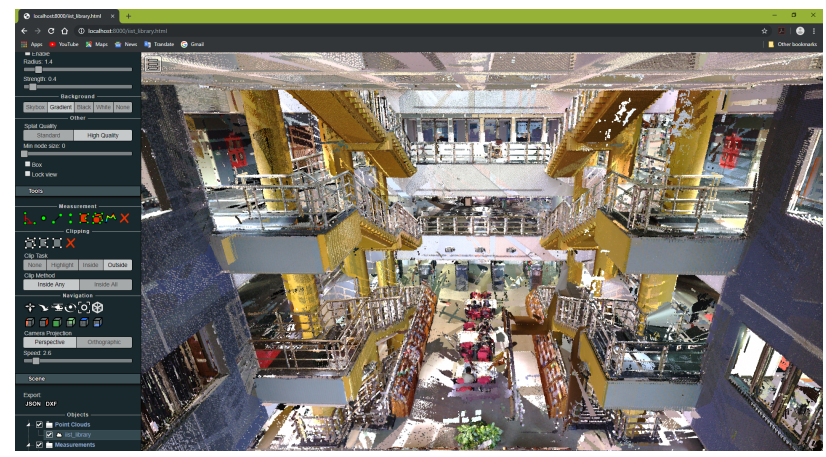

(b)

Figure 4. Visualisation of the interior of the library using Potree renderer.

In addition to visualisation, the renderer can also make basic measurements on the point cloud as shown in Figure 5 and Figure 6.

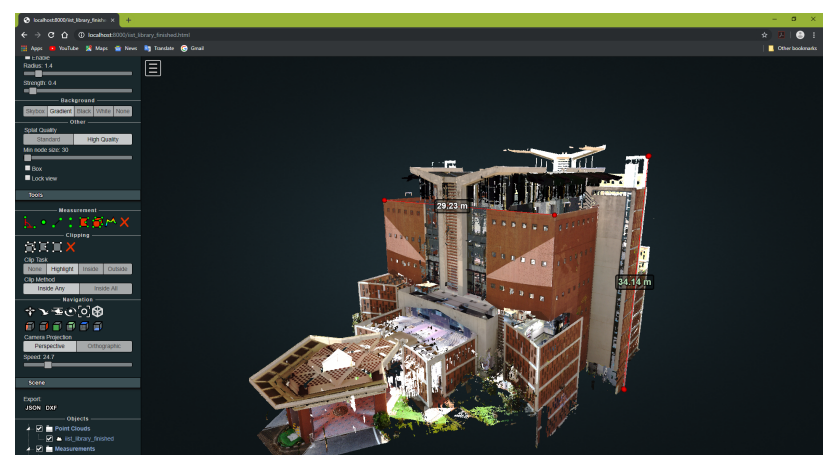

Figure 5. Potree facilitates basic measurements on the rendered point cloud.

The point cloud can also be visualised in false colour maps representing scalar fields (such as height) instead of true colour (RGB) as shown in Figure 7 and Figure 8.

Finally, it was observed that the dimensions of the point cloud measured from the Potree renderer showed good correlation with the actual dimensions of the structure.

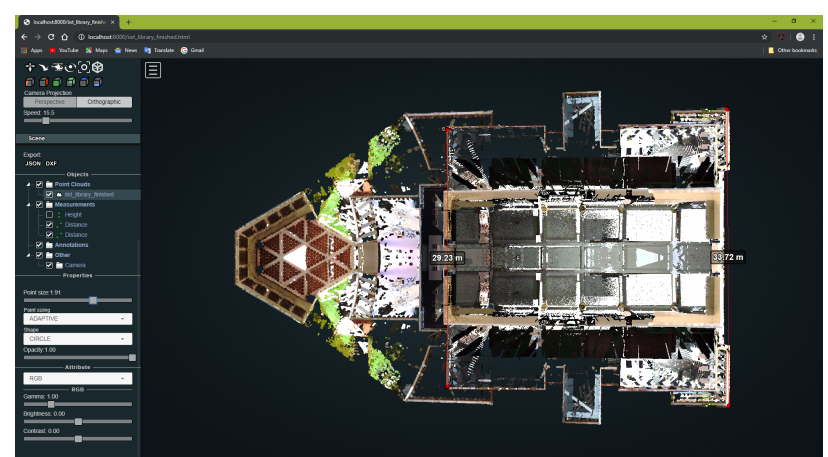

Figure 6. Top view of the library.

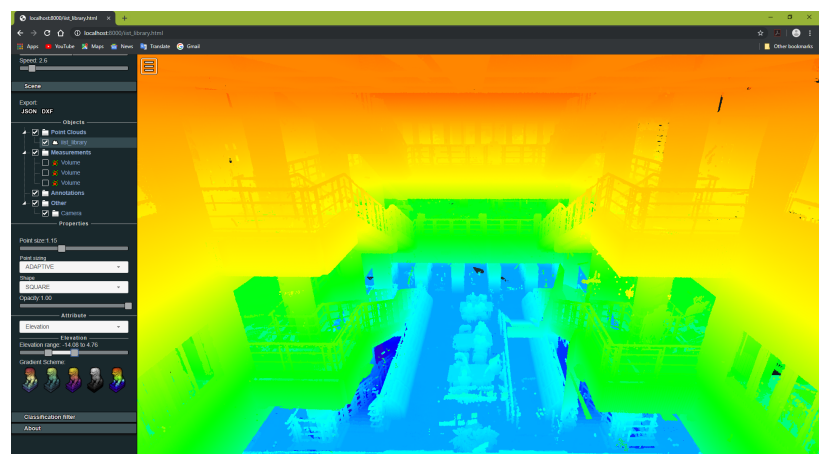

Figure 7. Interior of the library visualised in linear colour map representing height from ground.

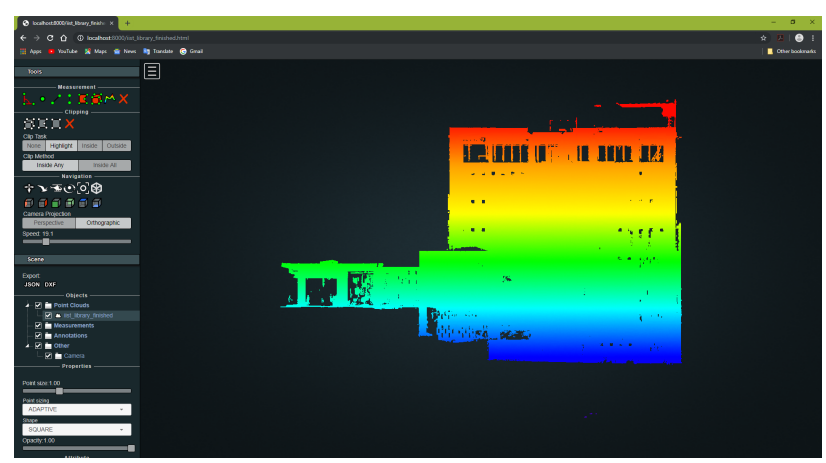

Figure 8. Exterior of the library visualised in linear colour map representing height from ground.

\subsection{DISCUSSIONS}

The Potree renderer is very much suited for the purpose of building information modelling (BIM) and 3D documentation of structures. It is both efficient and portable since it renders the point cloud in a WebGL based interface that makes it easy to share, distribute and publish. Furthermore, it supports real time rendering of the point cloud, due to which the loading times of the renderer are much lesser. The dimensions of the point cloud measured using the Potree renderer showed good correlation with the actual dimensions of the structure. This emphasises the feasibility of generating architectural models of the structure from the Potree renderer which could be the focus of a future study.

However, it should be noted that Potree relies heavily on the GPU while rendering. This could prove to be a drawback for users with legacy hardware and/or no GPUs. Furthermore, the 
Potree renderer is only able to visualise point clouds. Some BIMs would require the building to be visualised as a mesh or 3D object instead of a point cloud. This could prove to be a limitation for such cases. These could be seen as some of the present limitations of the Potree renderer at time of writing this paper. Further developing the application to overcome these limitations could also serve as topics of future research and development.

\section{ACKNOWLEDGEMENTS}

This work was carried out as part of our Master's degree coursework. We express our sincerest appreciation for the guidance and assistance provided by Dr. V. K. Dadhwal, Director, Indian Institute of Space Science and Technology (IIST). The access to the FARO Focus S 350 terrestrial laser scanner was provided by the Department of Earth and Space Sciences, IIST.

\section{REFERENCES}

Azhar, S., 2011. Building information modeling (BIM): Trends, benefits, risks, and challenges for the AEC industry. Leadership and Management in Engineering, 11(3), 241-252.

Eastman, C., Teicholz, P., Sacks, R., Liston, K., 2011. BIM Handbook: A Guide to Building Information Modeling for Owners, Managers, Designers, Engineers and Contractors. Wiley.

Fröhlich, C., Mettenleiter, M., 2004. Terrestrial laser scanning - New perpesctives in 3D surveying. International Archives of Photogrammetry, Remote Sensing, 36(8), 1-7.

Girardeau-Montaut, D., 2016. Cloudcompare.

Isenburg, M., 2012. LAStools-efficient tools for LiDAR processing. Available at: http: http://www. cs. unc. edu/ isenburg/lastools/[Accessed October 9, 2012].

Isenburg, M., 2013. LASzip: lossless compression of LiDAR data. Photogrammetric Engineering and Remote Sensing, 79(2), 209-217.

Kijewski-Correa, T., Gong, J., Kennedy, A., Womble, J., Cai, C., Cleary, J., Dao, T., Leite, F., Liang, D., Peterman, K., Sun, C., Taflanidis, A., Wood, R., 2018. Performance of low-rise construction under wind and coastal hazards during the landfall of hurricane harvey.

Pu, S., Vosselman, G., 2009. Knowledge based reconstruction of building models from terrestrial laser scanning data. ISPRS Journal of Photogrammetry and Remote Sensing, 64(6), 575584. http://dx.doi.org/10.1016/j.isprsjprs.2009.04.001.

Samberg, A., 2007. An implementation of the asprs las standard. ISPRS Workshop on Laser Scanning and SilviLaser, Citeseer, 363-372.

Schuetz, M., 2016. Potree: Rendering Large Point Clouds in Web Browsers. Master's thesis, Technische Universität Wien.

Schwarz, B., 2010. Lidar:
in 3D. Mapping the world http://dx.doi.org/10.1038/nphoton.2010.148.

\begin{abstract}
APPENDIX
A video demonstrating the functions of the application generated using Potree is available at the following URL: https://youtu.be/fBwMUnGnPR0
\end{abstract}

Revised October 2019 